Waves 8 Oscillations in the Solar Atmosphere:

Heating and Magneto-Seismology

Proceedings IAU Symposium No. 247, 2007

(c) 2008 International Astronomical Union

R. Erdélyi \&s C. A. Mendoza-Briceño, eds. doi:10.1017/S1743921308014841

\title{
Attenuation of non-adiabatic oscillations in a cartesian prominence fibril
}

\author{
R. Soler, R. Oliver and J. L. Ballester \\ Departament de Física, Universitat de les Illes Balears, E-07122, Palma de Mallorca, Spain \\ email: roberto.soler;ramon.oliver; joseluis.ballester@uib.es
}

\begin{abstract}
One of the typical features shown by observations of solar prominence oscillations is that they are quickly damped in time by one or several not well-known mechanisms. In addition, recent high resolution observations have revealed that the prominence fine structures, called fibrils, can oscillate with their own periods, independently from the rest of the prominence. The main aim of the present work is to study the attenuation of oscillations supported by a single prominence fibril. We consider an equilibrium made of a prominence plasma Cartesian slab of finite width embedded in a coronal medium, and assume non-adiabatic effects (thermal conduction, radiation losses and heating) as damping mechanisms. The magnetic field is taken uniform and parallel to the slab axis. We find that the efficiency of the non-adiabatic effects as damping mechanisms is different for each magnetoacoustic mode. The obtained values of the damping time are compatible with those observed in the case of the slow modes, but the fast modes are much less attenuated.
\end{abstract}

Keywords. Sun: oscillations - Sun: magnetic fields - Sun: corona - Sun: prominences

\section{Introduction}

The existence of waves and oscillations of small amplitude in solar quiescent prominences is a well-known phenomenon reported by a large number of observations. Observations usually detected oscillations of the whole or large areas of the prominence $(e . g$. Molowny-Horas et al. 1997; Terradas et al. 2002), but oscillations and motions of single filament threads have been also detected (e.g. Yi et al. 1991; Yi \& Engvold 1991; Lin et al. 2007). In addition, several observers (Molowny-Horas et al. 1999; Terradas et al. 2002) have also reported signs of a very quick attenuation of the oscillations. From the theoretical point of view, these oscillatory motions have often been interpreted in terms of magnetoacoustic wave modes (see Oliver \& Ballester 2002 for an extensive review). However, the mechanism responsible for the damping is still under investigation, and several candidates have been proposed (Ballai 2003). Taking into account simple prominence models, the damping mechanisms more extensively investigated to date are non-adiabatic effects, as radiation, thermal conduction and heating (Carbonell et al. 2004; Terradas et al. 2005), which seem to be only efficient in damping slow modes, whereas the fast waves remain almost undamped. On the other hand, Forteza et al. (2007) studied the role of the ion-neutral collisions on the time damping of oscillations in an unbounded prominence plasma, and found that the fast mode can be efficiently attenuated in almost neutral plasmas, but the slow wave is slightly influenced. Recently, Soler et al. (2007a) have studied the effect of non-adiabatic coronal mechanisms on the time damping of prominence oscillations, by considering a prominence model that includes the external corona. These authors found that the coronal thermal conduction have an important effect on the damping of the fast mode, which is more attenuated than in simple models that do not consider the presence of the corona. Nevertheless, the investigation of the time 
attenuation of vibrations supported by a single fibril, instead of the whole prominence, is still not performed. This is, then, the main motivation for the present work.

\section{Fibril Model and Basic Equations}

Our equilibrium configuration representing a prominence fibril (Fig. 1) is made of a homogeneous plasma layer with prominence conditions (density $\rho_{\mathrm{p}}=5 \times 10^{-11} \mathrm{~kg} \mathrm{~m}^{-3}$ and temperature $T_{\mathrm{p}}=8000 \mathrm{~K}$ ) embedded in an unbounded corona (density $\rho_{\mathrm{c}}=2.5 \times$ $10^{-13} \mathrm{~kg} \mathrm{~m}^{-3}$ and temperature $\left.T_{\mathrm{c}}=10^{6} \mathrm{~K}\right)$. The magnetic field is $\vec{B}_{0}=B_{0} \hat{e}_{x}$, with $B_{0}=5 \mathrm{G}$ everywhere. Both media are unlimited in the $x$ - and $y$-directions. The halfwidth of the slab is $z_{\mathrm{p}}=30 \mathrm{~km}$, whose value is motivated from the observations (Lin et al. 2005). This equilibrium have been studied by Edwin \& Roberts (1982), and recently revisited by Soler et al. (2007b), in the case of ideal, adiabatic perturbations. The reader is referred to these works for an extensive analysis of the eigenmodes supported by the model.

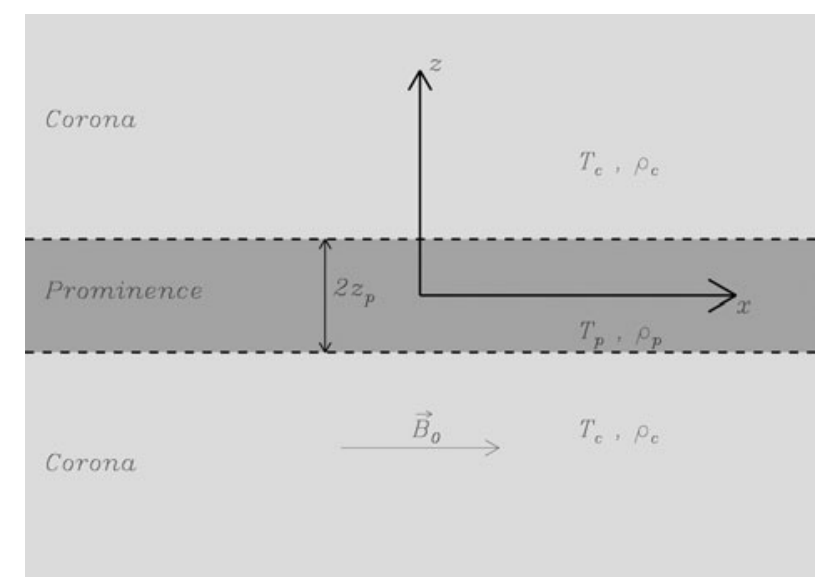

Figure 1. Sketch of the equilibrium configuration.

We consider parallel thermal conduction to the magnetic field, radiation and heating as non-ideal, damping mechanisms. Assuming small, linear perturbations from the equilibrium state and no propagation in the $y$-direction, the dispersion relation which governs the fast and slow non-adiabatic magnetoacoustic modes can be obtained by following the same process as in Soler et al. (2007a). This dispersion relation is then,

$$
\frac{\rho_{\mathrm{c}}}{\rho_{\mathrm{p}}}\left(k_{x}^{2} v_{\mathrm{Ac}}^{2}-\omega^{2}\right) k_{z \mathrm{p}}\left\{\begin{array}{l}
\cot \\
\tan
\end{array}\right\}\left(k_{z \mathrm{p}} z_{\mathrm{p}}\right) \pm\left(k_{x}^{2} v_{\mathrm{Ap}}^{2}-\omega^{2}\right) k_{z \mathrm{c}}=0,
$$

where the cot term and the + sign correspond to kink modes, whereas the tan term and the - sign correspond to sausage modes. $c_{\mathrm{s}}^{2}=\frac{\gamma p_{0}}{\rho_{0}}$ and $v_{\mathrm{A}}^{2}=\frac{B_{0}^{2}}{\mu \rho_{0}}$ are the adiabatic sound speed squared and the Alfvén speed squared, respectively, while subscripts p or c denote quantities computed using prominence or coronal values, respectively. In Eq. (2.1), $\omega=\omega_{\mathrm{R}}+i \omega_{\mathrm{I}}$ is the complex oscillatory frequency, $k_{x}$ is the wavenumber in the $x$-direction, and $k_{z}$ plays the role of the wavenumber in the $z$-direction and is given by

$$
k_{z}^{2}=\frac{\left(\omega^{2}-k_{x}^{2} v_{\mathrm{A}}^{2}\right)\left(\omega^{2}-k_{x}^{2} \Lambda^{2}\right)}{\left(v_{\mathrm{A}}^{2}+\Lambda^{2}\right)\left(\omega^{2}-k_{x}^{2} \tilde{c}_{\mathrm{T}}^{2}\right)},
$$

where $\Lambda^{2}$ and $\tilde{c}_{\mathrm{T}}^{2}$ are the modified sound and cusp (or tube) speed squared, respectively, 
due to the presence of non-adiabatic mechanisms,

$$
\begin{gathered}
\Lambda^{2} \equiv \frac{c_{\mathrm{s}}^{2}}{\gamma}\left[\frac{(\gamma-1)\left(\frac{T_{0}}{p_{0}} \kappa_{\|} k_{x}^{2}+\omega_{T}-\omega_{\rho}\right)+i \gamma \omega}{(\gamma-1)\left(\frac{T_{0}}{p_{0}} \kappa_{\|} k_{x}^{2}+\omega_{T}\right)+i \omega}\right], \\
\tilde{c}_{\mathrm{T}}^{2} \equiv \frac{v_{\mathrm{A}}^{2} \Lambda^{2}}{v_{\mathrm{A}}^{2}+\Lambda^{2}} .
\end{gathered}
$$

Finally, $\gamma$ is the adiabatic ratio, $\kappa_{\|}$is the parallel thermal conductivity to the magnetic field, and $\omega_{\rho}$ and $\omega_{T}$ are quantities related to the radiative losses and plasma heating (see Soler et al. 2007a for details).

Among all solutions of Eq. (2.1) for a fixed, real $k_{x}$, we focus to those evanescent in the corona $\left(\Re\left(k_{z \mathrm{c}}^{2}\right)<0\right)$ but body-like in the prominence $\left(\Re\left(k_{z \mathrm{p}}^{2}\right)>0\right)$. So, leaky waves $\left(\Re\left(k_{z \mathrm{c}}^{2}\right)>0\right)$ are discarded from this investigation. For simplicity, we also restrict ourselves to the fundamental kink modes. Then, we compute the period, $P$, the damping time, $\tau_{\mathrm{D}}$, and the ratio of both quantities as function to the real and imaginary parts of the frequency as follows,

$$
P=\frac{2 \pi}{\omega_{\mathrm{R}}}, \quad \tau_{\mathrm{D}}=\frac{1}{\omega_{\mathrm{I}}}, \quad \frac{\tau_{\mathrm{D}}}{P}=\frac{1}{2 \pi} \frac{\omega_{\mathrm{R}}}{\omega_{\mathrm{I}}}
$$

\section{Results and Discussion}

The results presented here correspond to an optically thin radiation and a constant heating per unit volume (see Soler et al. 2007a, Table 1). Figure 2 shows the period, $P$, the damping time, $\tau_{\mathrm{D}}$, and their ratio as function of $k_{x}$, corresponding to the fundamental kink modes.
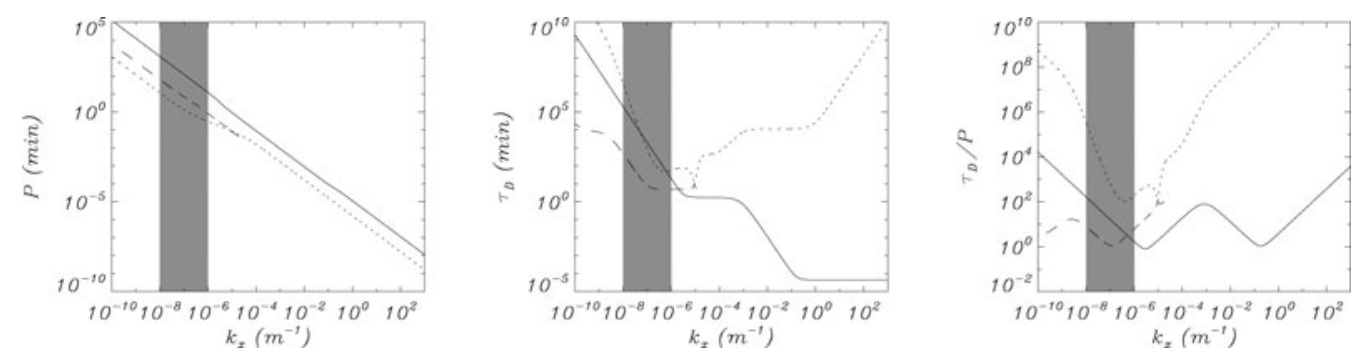

Figure 2. Period (left), damping time (centre) and ratio of the damping time to the period (right) versus the longitudinal wavenumber for the fundamental kink oscillatory modes: internal slow (solid line), fast (dotted line) and external slow (dashed line). The shaded zones correspond to those wavelengths typically observed in prominence oscillations.

Taking into account the results in the range of wavelengths typically observed in prominences, we see that the internal slow mode (responsible for longitudinal motions) produces periods compatible with intermediate- and large-period oscillations, whereas the fast mode (responsible for transverse motions) could be associated with short-period oscillations. On the other hand, the external slow mode mainly disturbs the surrounding corona, the amplitude of its motions within the prominence fibril being very small (Soler et al. 2007b), hence it could be rather difficult to observe. Regarding the damping time, both internal and external slow modes are efficiency attenuated, with damping times of the order of their periods. However, the fast wave is much less attenuated since its damping time is between 2 and 6 orders of magnitude larger than its period. This means that 
non-adiabatic effects are not efficient enough in damping transverse fibril oscillations, and so an additional mechanism (not considered here) is probably responsible for the observed damping times.

Now, in order to assess which is the relative importance of each non-adiabatic damping mechanism, we compare the damping time obtained when considering all non-adiabatic effects (displayed in the middle column of Fig. 2) with the results obtained when a specific mechanism is removed. With this analysis, we are able to find out where the omitted mechanism has an appreciable effect on the damping. The results of these computations are displayed in Fig. 3 and summarised next:
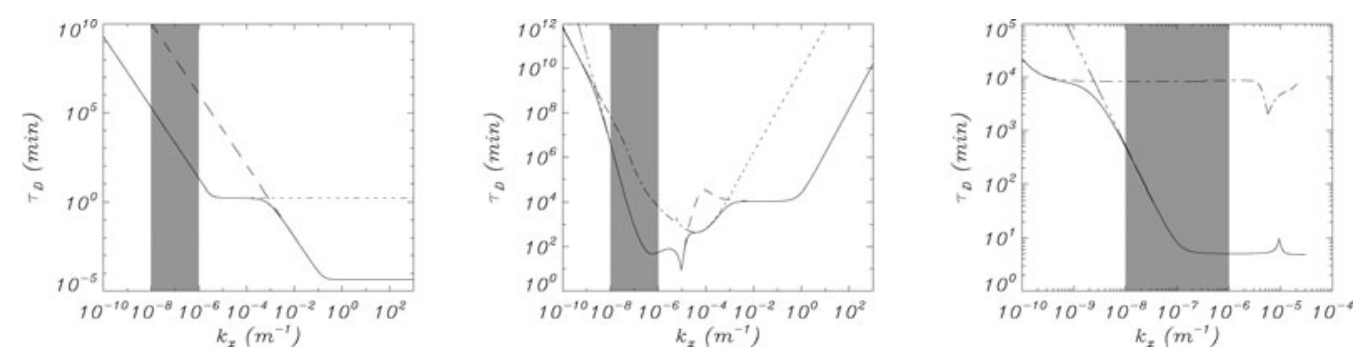

Figure 3. Damping time versus the longitudinal wavenumber for the three fundamental kink oscillatory modes: internal slow (left), fast (centre) and external slow (right). Different linestyles represent the omitted mechanism: all mechanisms considered (solid line), prominence conduction eliminated (dotted line), prominence radiation eliminated (dashed line), coronal conduction eliminated (dot-dashed line) and coronal radiation eliminated (three dot-dashed line).

- The internal slow mode is only affected by prominence-related mechanisms, radiative losses from the prominence plasma being responsible for the attenuation of this solution in the range of typically observed wavelengths. The prominence thermal conduction is only efficient for very small wavelengths (large $k_{x}$ ) outside the observed range.

- The fast mode is affected by both prominence and coronal mechanisms. The former are dominant for small wavenumber while the latter are more important for large $k_{x}$. The coronal conduction is the most important damping mechanism in the region of observed wavelengths. This involves an important novelty with respect to Soler et al. (2007a), in which a wider slab representing the whole prominence was considered, and the prominence radiation still had a relevant effect in the observed range of wavelengths. This means that the slab width has an important influence on value of $k_{x}$ that determines the transition between the regimes dominated by the prominence radiation and the coronal conduction.

- The damping of the external slow mode is entirely governed by coronal-related mechanisms, mainly the coronal thermal conduction, which is the dominant damping mechanism in the range of typically observed wavelengths.

\section{Conclusion}

In this work, we have performed a brief study on the time damping of the eigenmodes supported by a Cartesian prominence fibril. Radiative losses, plasma heating and parallel thermal conduction have been considered as damping mechanisms. In agreement with previous studies (Carbonell et al. 2004; Terradas et al. 2005), we have found that only the slow modes are efficiently damped by non-adiabatic effects. On the contrary, the efficiency of these mechanisms in damping transverse, fast-like oscillations is not enough to reproduce the observations. On the basis of this result, we think that another damping 
mechanism, much more efficient in damping transverse oscillations, should be responsible for the observed damping times. Some candidates could be resonant absorption in Alfvén or slow continua and ion-neutral collisions, whose effect on the damping of fibril oscillations should be investigated in further works.

\section{Acknowledgements}

The authors acknowledge the financial support received from the Spanish Ministerio de Ciencia y Tecnología and the Conselleria d'Economia, Hisenda i Innovació under Grant Nos. AYA2006-07637 and PCTIB-2005GC3-03, respectively. R. Soler thanks the Conselleria d'Economia, Hisenda i Innovació for a fellowship. The authors also thank the LOC in Isla de Margarita for their kind hospitality and for the excellent rum!

\section{References}

Ballai, I. 2003, A $\& A$, 410, L17

Carbonell, M., Oliver, R., \& Ballester, J. L. 2004, A\&A, 415, 739

Edwin, P.M. \& Roberts, B. 1982, Solar Phys., 76, 239.

Forteza, P., Oliver, R., Ballester, J. L., \& Khodachenko, M. L. 2007, A\&A, 461, 731

Lin, Y., Engvold, O., Rouppe van der Voort, L. H. M. et al. 2005, Solar Phys., 226, 239

Lin, Y., Engvold, O., Rouppe van der Voort, L. H. M., \& van Noort, M. 2007, Solar Phys., in press. DOI:10.1007/s11207-007-0402-8

Molowny-Horas, R., Oliver, R., Ballester, J. L., \& Baudin, F. 1997, Solar Phys., 172, 181

Molowny-Horas, R., Wiehr, E., Balthasar, H. et al. 1999, JOSO Annual Report 1998, Astronomical Institute Tatranska Lomnica, 126

Oliver, R. \& Ballester, J. L. 2002, Solar Phys., 206, 45

Soler, R., Oliver, R., \& Ballester, J. L. 2007a, A\&A, 471, 1023

Soler, R., Oliver, R., \& Ballester, J. L. 2007b, Solar Phys., in press. DOI:10.1007/s11207-0079084-5

Terradas, J., Molowny-Horas, R., Wiehr, E. et al. 2002, A\& A, 393, 637

Terradas, J., Carbonell, M., Oliver, R., \& Ballester, J. L. 2005, A\& A, 434, 741

Yi, Z., Engvold, O., \& Kiel, S. L. 1991, Solar Phys., 132, 63

Yi, Z. \& Engvold, O. 1991, Solar Phys., 134, 275 